\title{
Ureter Abscess
}

National Cancer Institute

\section{Source}

National Cancer Institute. Ureter Abscess. NCI Thesaurus. Code C35666.

An abscess that is located in the ureter. 